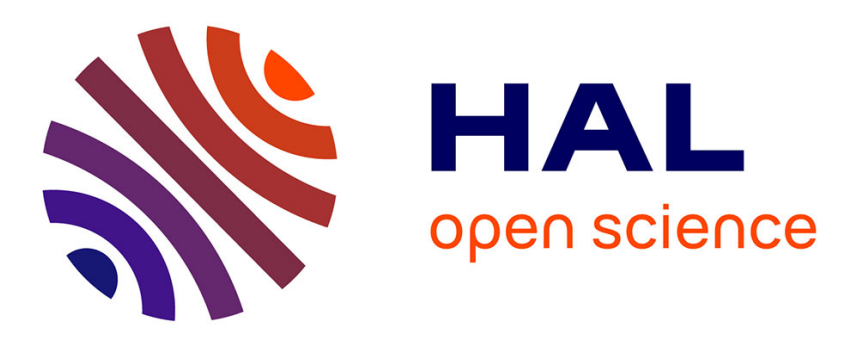

\title{
Improved localization of near-field sources using a realistic signal propagation model and optimally-placed sensors
}

Jean-Pierre Delmas, Houcem Gazzah, Mohamed Nabil El Korso

\section{To cite this version:}

Jean-Pierre Delmas, Houcem Gazzah, Mohamed Nabil El Korso. Improved localization of near-field sources using a realistic signal propagation model and optimally-placed sensors. Digital Signal Processing, 2019, 95, pp.102579-1 - 102579-9. 10.1016/j.dsp.2019.102579 . hal-02387919

\section{HAL Id: hal-02387919 \\ https://hal.science/hal-02387919}

Submitted on 30 Nov 2019

HAL is a multi-disciplinary open access archive for the deposit and dissemination of scientific research documents, whether they are published or not. The documents may come from teaching and research institutions in France or abroad, or from public or private research centers.
L'archive ouverte pluridisciplinaire HAL, est destinée au dépôt et à la diffusion de documents scientifiques de niveau recherche, publiés ou non, émanant des établissements d'enseignement et de recherche français ou étrangers, des laboratoires publics ou privés. 


\title{
Improved localization of near-field sources using a realistic signal propagation model and optimally-placed sensors
}

\author{
Jean-Pierre Delmas ${ }^{\mathrm{a}}$, Houcem Gazzah ${ }^{\mathrm{b}}$, Mohammed Nabil El Korso ${ }^{\mathrm{c}}$ \\ ${ }^{a}$ Telecom SudParis, Departement CITI, CNRS UMR 5157, Evry, France, E-mail: jean-pierre.delmas@it-sudparis.eu \\ ${ }^{b}$ Department of Electrical and Computer Engineering, University of Sharjah, 27272, UAE, E-mail: hgazzah@sharjah.ac.ae \\ ${ }^{c}$ Université Paris Nanterre, LEME laboratory EA 4416, France, E-mail: m.elkorso@parisnanterre.fr
}

\begin{abstract}
In this paper we analyze the estimation of the angle and the range of a narrow-band source located in the near-field of an arbitrary centro-symmetric linear array (CSLA). This analysis deals with the Cramer Rao bound $(\mathrm{CRB})$ on both angle and range, obtained thanks to an exact expression of the source-to-sensor delay and a realistic (range-dependent) model of source-to-sensor attenuation, ultimately achieving two objectives. On the first hand, closed-form approximate expressions of the CRB are developed and compared to those obtained assuming (unrealistically) that sensors perceive the same power despite being at different distances from the source. While the impact on angle estimation is negligible, range CRB significantly decreases if one incorporates the more appropriate range-dependent power model (except for sources at broadsides). An important consequence is that localization algorithms taking this range-dependent modelization of the apparent source power into account in their signal modeling should have much better range performance. On the second hand, the obtained CRBs are used to design nonuniform CSLA taking into account the ambiguities, with improved angle and range estimation, comparatively to uniform linear arrays (ULA). Finally, we show that our optimized CSLA for a single source also brings some benefits for two closelyspaced sources.
\end{abstract}

Keywords: Cramer Rao bounds, linear antenna arrays, power profile, direction-of-arrival and range estimation, near-field source localization, array optimization.

\section{Introduction}

CRBs are usually used to benchmark parameter estimation algorithms. Furthermore, if interpretable expressions are obtained, they can be used to optimize the system design, for instance, to minimize the variance of the estimated parameters (see e.g., [1, 2]). In the particular context of source localization, much effort has been made to the far-field case for decades (see e.g., [3, 4, 5] and references therein) where the distance of the source to the array is large compared with the array aperture, and hence the propagating waves are considered to be plane waves at the sensor array and only the source direction of arrival (DOA) can be estimated. 
It is possible to estimate the range (distance from the source to the array) if this distance is not too large compared to the array aperture. This near-field situation occurs in many practical applications such as sonar [6], speaker localization [7, electronic surveillance [8, object detection 9], collision avoidance radar [10], robot navigation [11], sismic exploration [12], biomedical imaging [13], 14], seismic exploration [15], etc. In this near-field case, wavefronts are spherical and received power varies from sensor to sensor. However, to reduce the complexity of the localization algorithms, an approximate propagation model relevant to the so-called Fresnel zone has been used. This latter makes use of the second-order Taylor expansion of the time delay parameter, with constant amplitude gain however. Numerous methods have used these approximations, such as a polynomial rooting approach [16], an high-order ESPRIT algorithm [17], a weighted linear prediction method [18, an ESPRIT/MUSIC procedure exploiting subarrays [19], a two-stage MUSIC algorithm [20], a least-square procedure [21], a prediction and oblique projection operator method [22] and many other approaches. Furthermore, these approximations facilitate the CRB derivations (see e.g., 23]).

Only lately the exact time delay and range-dependent modelization of the apparent source power (called also power profile) have been used [24, but only to derive a complicated non-interpretable approximate expression of the near-field CRB for the ULA case. We consider here arbitrary CSLA made of pairs of sensors symmetrically located along the two sides of the linear antenna array. Such class of nonuniform linear arrays are chosen for their attractive features proved in [25] for constant amplitude gains. This includes lower DOA and range CRBs and faster convergence to the lower far-field DOA CRB. Furthermore, thanks to the decoupling between the DOA and range parameters to the second-order w.r.t. the inverse of the range in the Fisher information matrix, the derivation of closed-form approximate expressions of the CRB is greatly simplified. Note that we use a definition of the near-field that is familiar in the signal processing literature, designating the region where range estimation makes sense (to be distinguished from the reactive and radiative region, as understood in electromagnetism [26, ch.2].

In this paper, we first develop interpretable and accurate closed-form approximate expressions of the CRB for both source angle and range. They are compared to those unaware of dependence of received power on source range. These expressions are proved to depend only on three geometric parameters only: the second, fourth and sixth-order moments of the positions of the sensors forming the arbitrary but centro-symmetric linear array (CSLA). The obtained expressions tend to prove that the CRB on the angle is generally barely impacted by the power profile. In contrast, the CRB on the range is strongly reduced, except at broadside directions, i.e. almost everywhere. Second, thanks to these closed-form expressions we design nonuniform CSLA with improved range estimation (by as much as 60\%) with identical CRB on the angle with respect to ULAs. This design also incorporates geometric constraints to account for the array ambiguity problem. Specifically, these constraints lead to a constrained max-min problem. We use its equivalent to a global polynomial maximization under, both polynomial equalities and inequalities which can be efficiently solved using the Matlab GloptiPoly utility [36. Finally, we show that our optimized CSLA for a single source also 
brings some benefits for both DOA and range estimation in the context of two closely-spaced sources.

The paper is organized as follows. Section 2 introduces the data model. After giving the general expression of the deterministic and stochastic CRB concentrated on the localization parameters, we develop an interpretable closed-form approximate expressions of the CRB on both angle and range in Section 3 Section 4 is dedicated to analytical comparisons of these CRBs to the CRBs not taking the power profile into account. These closed-form expressions are used in Section 5 to design nonuniform CSLA with improved range estimation and immunity against array ambiguities. Finally, a conclusion is given in Section 6 .

\section{Data Model}

We consider a linear (possibly nonuniform) antenna array made of $P$ sensors $C_{1}, \cdots, C_{P}$ depicted in Fig.1, located along a straight line at coordinates $x_{1}, \cdots, x_{P}$, respectively. Without loss of generality, we assume the array centroid to be at the origin $O$ of this axis. This choice allows for more compact expressions of the CRB compared to [23, 24].

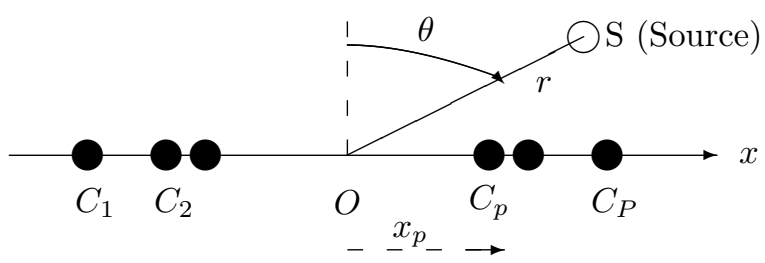

Fig.1 Source in the near-field impinging on an arbitrary linear array.

A source is located at point $S$, at a range $r$ from the plane origin $O$, and forming an angle $\theta$ w.r.t. the axis perpendicular to the array. This single source is emitting a narrow band signal of wavelength $\lambda$ with no multipath so that the complex baseband snapshot collected by the sensor $p$ at time index $t$ reads

$$
y_{p}(t)=g_{p} e^{i \tau_{p}} s(t)+n_{p}(t)
$$

where $s(t)$ and $n_{p}(t)$ represent, respectively, the source signal collected at the origin and the ambient additive noise collected by sensor $p$. The exact expression of the phase $\tau_{p}$ is defined as $\tau_{p}=2 \pi\left(S O-S C_{p}\right) / \lambda$. Using the law of cosine, it is rewritten as

$$
\tau_{p}=2 \pi \frac{r}{\lambda}\left(1-\sqrt{\beta_{p}}\right)
$$

with

$$
\beta_{p} \stackrel{\text { def }}{=} 1-2 \frac{x_{p}}{r} \sin \theta+\frac{x_{p}^{2}}{r^{2}} .
$$

Note that, because we fix the phase and amplitude references at the centroid of the array, our definition of the tuple $(\theta, r)$ is different from the one in [24, which fixes the phase reference at the first sensor. Regarding the gain $g_{p}$ at sensor $p$, we assume a spherical wavefront and a specific range-dependent power profile, where 
the signal magnitude is inversely proportional to the distance from the source [26, Chap.2]:

$$
g_{p}=\frac{S O}{S C_{p}}=\frac{1}{\sqrt{\beta_{p}}}
$$

Thus the sensed power is variable from sensor to sensor. Again, because we use the origin as reference, our definition of $g_{p}$ is different from the one used [24, for which the gain is not defined with respect to a reference: $g_{p}=\frac{1}{S C_{p}}=\frac{1}{r \sqrt{\beta_{p}}}$.

We collect $N$ snapshots $\left\{y_{p}(t)\right\}_{p=1, \ldots, P ; t=t_{1}, \ldots, t_{N}}$, to estimate both angle $\theta$ and range $r$. Estimation accuracy is evaluated in terms of the CRB, developed under the following commonly used assumptions about signal and noise [27]:

(i) $n_{p}(t)$ and $s(t)$ are independent,

(ii) $\left\{n_{p}(t)\right\}_{p=1, . ., P ; t=t_{1}, . ., t_{N}}$ are independent, zero-mean circular Gaussian distributed with variance $\sigma_{n}^{2}$,

(iii) $\{s(t)\}_{t=t_{1}, . ., t_{N}}$ are assumed to be either deterministic unknown parameters (the so-called conditional or ${ }_{65}$ deterministic model) with $\sigma_{s}^{2}=\frac{1}{N} \sum_{n=1}^{N}\left|s\left(t_{n}\right)\right|^{2}$, or independent zero-mean circular Gaussian distributed with variance $\sigma_{s}^{2}$ (the so-called unconditional or stochastic model).

\section{Expressions of the CRB}

\subsection{Theoretical general background on CRB for near-field sources}

We focus on a single near-field source whose location is characterized by the parameter of interest

$$
\boldsymbol{\alpha}=[\theta, r]^{T} .
$$

When the sensed power is constant across all sensors, stochastic and deterministic matrix-valued CRBs (concentrated on the parameter of interest) are equal, up to a multiplicative term depending only on the SNR $\sigma_{s}^{2} / \sigma_{n}^{2}$ of the source and the number $P$ of sensors [25. This contrasts with the case where the power profile is taken into account for which the multiplicative term depends on $\boldsymbol{\alpha}$ :

$$
\mathrm{CRB}_{\text {sto }}(\boldsymbol{\alpha})=\left(1+\frac{\sigma_{n}^{2}}{\|\mathbf{a}(\boldsymbol{\alpha})\|^{2} \sigma_{s}^{2}}\right) \mathrm{CRB}_{\operatorname{det}}(\boldsymbol{\alpha}),
$$

where $\mathbf{a}(\boldsymbol{\alpha})$ is the steering vector of components $g_{p} e^{i \tau_{p}}, p=1, \ldots, P$. Obviously, the expression of the stochastic CRB can no longer be decoupled in power and geometric terms. Instead, these CRBs are given by the following expressions:

$$
\mathrm{CRB}_{\text {sto }}(\boldsymbol{\alpha})=c_{\sigma}^{\text {sto }}(\boldsymbol{\alpha}) \mathbf{F}^{-1}(\boldsymbol{\alpha}) \text { and } \mathrm{CRB}_{\operatorname{det}}(\boldsymbol{\alpha})=c_{\sigma}^{\mathrm{det}}(\boldsymbol{\alpha}) \mathbf{F}^{-1}(\boldsymbol{\alpha})
$$

where both CRBs appear to be inversely proportional to the matrix

$$
\mathbf{F}(\boldsymbol{\alpha})=\operatorname{Re}\left[\|\mathbf{a}(\boldsymbol{\alpha})\|^{2} \mathbf{D}^{H}(\boldsymbol{\alpha}) \mathbf{D}(\boldsymbol{\alpha})-\mathbf{D}^{H}(\boldsymbol{\alpha}) \mathbf{a}(\boldsymbol{\alpha}) \mathbf{a}^{H}(\boldsymbol{\alpha}) \mathbf{D}(\boldsymbol{\alpha})\right],
$$


through the constants

$$
c_{\sigma}^{\text {sto }}(\boldsymbol{\alpha}) \stackrel{\text { def }}{=} \frac{\sigma_{n}^{2}\left(\sigma_{n}^{2}+\|\mathbf{a}(\boldsymbol{\alpha})\|^{2} \sigma_{s}^{2}\right)}{2 N \sigma_{s}^{4}} \text { and } c_{\sigma}^{\text {det }}(\boldsymbol{\alpha}) \stackrel{\text { def }}{=} \frac{\|\mathbf{a}(\boldsymbol{\alpha})\|^{2} \sigma_{n}^{2}}{2 N \sigma_{s}^{2}}
$$

where $\mathbf{D}(\boldsymbol{\alpha})$ is defined as $\left[\frac{\partial \mathbf{a}(\boldsymbol{\alpha})}{\partial \theta}, \frac{\partial \mathbf{a}(\boldsymbol{\alpha})}{\partial r}\right]$. We note that whenever $P \sigma_{s}^{2} \gg \sigma_{n}^{2}$, we have

$$
c_{\sigma}^{\text {sto }}(\boldsymbol{\alpha}) \approx c_{\sigma}^{\operatorname{det}}(\boldsymbol{\alpha})
$$

The above condition means that the source is more powerful than ambient noise, which is more relevant to near-field sources. We maintain this assumption and realize that the stochastic CRB reduces to the deterministic CRB, on which we focus our attention from now on. In this case, the elements of the $2 \times 2$ matrix $\mathbf{F}(\boldsymbol{\alpha})$ given in 8 reads

$$
\begin{aligned}
{[\mathbf{F}]_{i, j} } & =\left(\sum_{p=1}^{P} g_{p}^{2}\right)\left(\sum_{p=1}^{P} g_{p, i}^{\prime} g_{p, j}^{\prime}+\tau_{p, i}^{\prime} \tau_{p, j}^{\prime} g_{p}^{2}\right) \\
& -\left(\sum_{p=1}^{P} g_{p, i}^{\prime} g_{p}\right)\left(\sum_{p=1}^{P} g_{p, j}^{\prime} g_{p}\right)-\left(\sum_{p=1}^{P} \tau_{p, i}^{\prime} g_{p}^{2}\right)\left(\sum_{p=1}^{P} \tau_{p, j}^{\prime} g_{p}^{2}\right), \quad i=1,2
\end{aligned}
$$

where $g_{p, 1}^{\prime} \stackrel{\text { def }}{=} \frac{\partial g_{p}}{\partial \theta}, g_{p, 2}^{\prime} \stackrel{\text { def }}{=} \frac{\partial g_{p}}{\partial r}, \tau_{p, 1}^{\prime} \stackrel{\text { def }}{=} \frac{\partial \tau_{p}}{\partial \theta}$ and $\tau_{p, 2}^{\prime} \stackrel{\text { def }}{=} \frac{\partial \tau_{p}}{\partial r}$.

\subsection{Taylor expansion of the matrix $\mathbf{F}(\boldsymbol{\alpha})$}

To highlight the impact of the power profile on the localization performance, we use, similarly to [25], a Taylor expansion of the matrix $\mathbf{F}(\boldsymbol{\alpha})$. To this end, we consider arbitrary CSLA, where for each sensor placed at coordinate $x_{p}$, there exists a symmetric sensor placed at coordinate $-x_{p}$. As a consequence, sums of the form $\sum_{p=1}^{P} x_{p}^{2 k+1}, k=0,1, \ldots$ are zero, while constants $S_{2 k} \stackrel{\text { def }}{=} \sum_{p=1}^{P} x_{p}^{2 k}$, characterizing the array geometry, will appear in the following Taylor expansion of the matrix $\mathbf{F}(\boldsymbol{\alpha})$. After tedious algebraic manipulations (main steps are shown in Appendix 7.1), we obtain:

$$
\begin{aligned}
\frac{\lambda_{\pi}^{2}}{r^{2} \cos ^{2} \theta}[\mathbf{F}(\boldsymbol{\alpha})]_{1,1}= & P \frac{S_{2}}{r^{2}}+\frac{P \lambda_{\pi}^{2} S_{2}+2 P S_{4}\left(6 \sin ^{2} \theta-1\right)-S_{2}^{2}\left(1+5 \sin ^{2} \theta\right)}{r^{4}}+\varnothing\left(r^{-4}\right), \\
\frac{2 \lambda_{\pi}^{2}}{r \sin \theta \cos \theta}[\mathbf{F}(\boldsymbol{\alpha})]_{1,2} & =\frac{-2 P \lambda_{\pi}^{2} S_{2}+\left(5 P S_{4}-3 S_{2}^{2}\right) \cos ^{2} \theta}{r^{4}}+o\left(r^{-4}\right), \\
\lambda_{\pi}^{2}[\mathbf{F}(\boldsymbol{\alpha})]_{2,2} & =\frac{4 P \lambda_{\pi}^{2} S_{2} \sin ^{2} \theta+\left(P S_{4}-S_{2}^{2}\right) \cos ^{4} \theta}{4 r^{4}} \\
& +\frac{\lambda_{\pi}^{2}\left[P S_{4}\left(1-15 \sin ^{2} \theta+24 \sin ^{4} \theta\right)-S_{2}^{2}\left(1-7 \sin ^{2} \theta+12 \sin ^{4} \theta\right)\right]}{r^{6}} \\
& +\frac{P S_{6}\left(-5+57 \sin ^{2} \theta-99 \sin ^{4} \theta+47 \sin ^{6} \theta\right)}{8 r^{6}} \\
& +\frac{S_{2} S_{4}\left(5-49 \sin ^{2} \theta+83 \sin ^{4} \theta-39 \sin ^{6} \theta\right)}{8 r^{6}}+\varnothing\left(r^{-6}\right),
\end{aligned}
$$

75 where $\lim _{\epsilon \rightarrow 0} O(\epsilon) / \epsilon=0$ with $x_{1}, \ldots x_{P}$ and $\lambda$ fixed and $\lambda_{\pi} \stackrel{\text { def }}{=} \frac{\lambda}{2 \pi}$. These expressions are to be compared to the expressions [25, rels. (7-9)] which can be retrieved from 10 by setting $g_{p, i}^{\prime}=0, i=1,2$ to account 
for constant gain. Compared to [25, rels. (7-9)], [11), (12) and (13) are much more intricate because they include the power profile. Note that for more general profiles (i.e., $g_{p}=\left(\frac{S O}{S C_{p}}\right)^{\alpha}$ with $\alpha \neq 1$ ), the derivation of (11), 120 and (13) from (10) would give even more complicated expressions difficult to exploit.

\subsection{Taylor expansion of $C R B(\theta)$ and $C R B(r)$ and key geometric parameters}

In the process of deriving the new CRB expressions, we identify the following three key geometric parameters $\left(S_{2}, \kappa, \eta\right)$ where

$$
\kappa \stackrel{\text { def }}{=} \frac{P S_{4}}{S_{2}^{2}} \text { and } \eta \stackrel{\text { def }}{=} \frac{P^{2} S_{6}}{S_{2}^{3}}
$$

play an important role in the array processing performance as well the antenna design. Using results (11)(13) and (7) in which we replace

$$
\|\mathbf{a}(\boldsymbol{\alpha})\|^{2}=P\left(1-\frac{S_{2}}{P r^{2}}\left(1-4 \sin ^{2} \theta\right)+o\left(r^{-2}\right)\right),
$$

we prove in the Appendix 7.2 , the following second-order expansions:

$$
\begin{aligned}
\operatorname{CRB}(\theta) & =\frac{c \lambda_{\pi}^{2}}{P S_{2} \cos ^{2} \theta}\left[1+\frac{\left(\frac{2 \kappa S_{2}}{P}-\lambda_{\pi}^{2}\right)+\frac{\sin ^{2} \theta}{P} g\left(\sin ^{2} \theta, S_{2}, \lambda_{\pi}^{2}, \kappa\right)}{r^{2}}+o\left(r^{-2}\right)\right], \\
\frac{\mathrm{CRB}(r)}{r^{4}} & =\frac{4 c \lambda_{\pi}^{2}}{S_{2}^{2}\left(4 P \frac{\lambda_{\pi}^{2}}{S_{2}} \sin ^{2} \theta+(\kappa-1) \cos ^{4} \theta\right)}\left[1+\frac{h\left(\sin ^{2} \theta, S_{2}, \lambda_{\pi}^{2}, \kappa, \eta\right)}{r^{2}}+o\left(r^{-2}\right)\right],
\end{aligned}
$$

where

$$
c \stackrel{\text { def }}{=} \frac{P}{2 N} \frac{\sigma_{n}^{2}}{\sigma_{s}^{2}}
$$

and

$$
g\left(\sin ^{2} \theta, S_{2}, \lambda_{\pi}^{2}, \kappa\right)=4 P^{2} \lambda_{\pi}^{4}+S_{2}^{2} \kappa(13 \kappa-9) \cos ^{4} \theta+4 P S_{2} \lambda_{\pi}^{2}\left[(6-7 \kappa) \sin ^{2} \theta-(5 \kappa-3)\right]
$$

and where $h\left(\sin ^{2} \theta, S_{2}, \lambda_{\pi}^{2}, \kappa, \eta\right)$ is an intricate function given in the Appendix 7.2 . We note that its expression is not useful to give a good numerical approximation of $\operatorname{CRB}(r)$ as it will be shown in Fig.3, where the approximate value deduced from (17) and (23) is very close to the approximate value deduced from only the ratio of the dominant terms of (17) and (23) given by (27).

\section{Analysis of the CRB}

\subsection{Far-field vs near-field performance}

We recall the deterministic far-field DOA CRB for arbitrary linear arrays [28, rel. (7)]

$$
\mathrm{CRB}_{\mathrm{FF}}(\theta)=\frac{1}{N} \frac{\lambda^{2}}{8 \pi^{2} S_{2} \cos ^{2} \theta} \frac{\sigma_{n}^{2}}{\sigma_{s}^{2}},
$$

allowing one to rewrite 16 as follows

$$
\operatorname{CRB}(\theta)=\operatorname{CRB}_{\mathrm{FF}}(\theta)\left[1+\frac{\left(\frac{2 \kappa S_{2}}{P}-\lambda_{\pi}^{2}\right)+\frac{\sin ^{2} \theta}{P} g\left(\sin ^{2} \theta, S_{2}, \lambda_{\pi}^{2}, \kappa\right)}{r^{2}}+o\left(r^{-2}\right)\right],
$$

which is consistent with 20 for $r$ tending to infinity where the effect of the power profile disappears. 


\subsection{Constant gain vs range and angle-dependent gain}

On the one side we have the near-field angle (16) and range (17) CRBs. On the other side we have the near-field angle $\mathrm{CRB}_{\mathrm{CG}}(\theta)$ and range $\mathrm{CRB}_{\mathrm{CG}}(r)$ assuming constant gain (not taking in account the power profile, i.e., $\left.g_{p}=1\right)$. The former are given by [25, rels. (15-16)]:

$$
\begin{aligned}
\operatorname{CRB}_{\mathrm{CG}}(\theta) & =\frac{c \lambda_{\pi}^{2}}{P S_{2} \cos ^{2} \theta}\left[1+\frac{\kappa S_{2}}{P r^{2}}\left\{1+\left(1+\frac{4 \kappa}{\kappa-1}\right) \sin ^{2} \theta\right\}+o\left(r^{-2}\right)\right], \\
\frac{\mathrm{CRB}_{\mathrm{CG}}(r)}{r^{4}} & =\frac{4 c \lambda_{\pi}^{2}}{S_{2}^{2}(\kappa-1) \cos ^{4} \theta}\left[1+k\left(\sin ^{2} \theta, \kappa, \eta\right) \frac{S_{2}}{P r^{2}}+o\left(r^{-2}\right)\right],
\end{aligned}
$$

where

$$
k\left(\sin ^{2} \theta, \kappa, \eta\right) \stackrel{\text { def }}{=} \frac{\left(2+18 \kappa^{2}+3 \kappa-23 \eta\right) \sin ^{2} \theta+3(\eta-\kappa)}{\kappa-1} .
$$

Obviously, the dominant terms $\frac{c \lambda_{\pi}^{2}}{P S_{2} \cos ^{2} \theta}$ of 16 and 22 are equal, implying that the near-field DOA CRB is barely affected by the power profile for ranges that are not too small. To go further, we look into the second-order term (in $1 / r^{2}$ ) in 16 and 22 . For example for $\theta=0$, we get:

$$
\frac{\operatorname{CRB}(\theta)_{\mid \theta=0}}{\operatorname{CRB}_{\mathrm{CG}}(\theta)_{\mid \theta=0}}=1+\frac{1}{r^{2}}\left(\frac{\kappa S_{2}}{P}-\lambda_{\pi}^{2}\right)+o\left(r^{-2}\right),
$$

95

where $\frac{\kappa S_{2}}{P}-\lambda_{\pi}^{2}$ is in practice positive. Indeed $\kappa \geq 1[25]$ implies that $\frac{\kappa S_{2}}{P}-\lambda_{\pi}^{2} \geq \frac{1}{P} \sum_{p=1}^{P} x_{p}^{2}-\frac{\lambda^{2}}{4 \pi^{2}}$ and the condition $\frac{1}{P} \sum_{p=1}^{P} x_{p}^{2}>\frac{\lambda^{2}}{4 \pi^{2}}$ is in practice satisfied given the non-ambiguity and aperture constraints.

For example, for a ULA with half-wavelength spacing and $P=2 Q$,

$$
\frac{1}{P} \sum_{p=1}^{P} x_{p}^{2}=\frac{\lambda^{2}\left(4 Q^{2}-1\right)}{48}>\frac{\lambda^{2}}{4 \pi^{2}} \text { from } Q=1 .
$$

Consequently $\operatorname{CRB}(\theta)$ is slightly larger than $\operatorname{CRB}_{\mathrm{CG}}(\theta)$ for broadside directions (i.e., $\theta \approx 0$ ).

A similar comparison of the near-field range $\mathrm{CRB}$ is expressed by the following ratio of the dominant terms of 17 and 23 for arbitrary angle $\theta$

$$
\frac{\mathrm{CRB}(r)}{\mathrm{CRB}_{\mathrm{CG}}(r)}=\left(1+\frac{4 P \lambda_{\pi}^{2}}{S_{2}} \frac{\sin ^{2} \theta}{(\kappa-1) \cos ^{4} \theta}\right)^{-1}\left(1+o\left(r^{-1}\right)\right) .
$$

From the above, the dominant term of $\mathrm{CRB}(r)$ is always smaller than the dominant term of $\mathrm{CRB}_{\mathrm{CG}}(r)$, except for $\theta=0$, for which they are equal. In particular for array end-fire directions (i.e., $|\theta| \approx \pi / 2), \operatorname{CRB}(r)$ is much lower than $\mathrm{CRB}_{\mathrm{CG}}(r)$. Consequently, taking into account this power profile allows one to achieve better range estimation without deteriorating angle estimation. This is explained by a larger sensitivity of the gain to the range with respect to the angle, for the end-fire directions. Furthermore, for these directions the time delay profile is less sensitive than the power profile for the range.

These results are confirmed in Figs. 2 and 3 which show respectively the ratios $\operatorname{CRB}(\theta) / \mathrm{CRB}_{\mathrm{CG}}(\theta)$ and $\operatorname{CRB}(r) / \mathrm{CRB}_{\mathrm{CG}}(r)$, as a function of the angle $\theta \in[0, \pi / 2)$. There, we assume a ULA of 6 sensors with half-wavelength inter-sensors spacing for a source at range $r=10 \lambda$. We see, in particular, that 
$\operatorname{CRB}(\theta) / \operatorname{CRB}_{\mathrm{CG}}(\theta) \in[0.83,1.03]$, whereas $\operatorname{CRB}(r) / \mathrm{CRB}_{\mathrm{CG}}(r)$ strongly decreases in $[0, \pi / 2)$, taking values $1,0.3$ and 0.005 for $\theta=0,60^{\circ}$ and $80^{\circ}$, respectively. These figures also show a good agreement between the approximate expressions of the CRBs deduced from (16), 17) and 22, , 23) and the exact ones (deduced from the exact expression of the matrix $\mathbf{F}(7),(8)$ and $(10)$. Furthermore, we see in Fig.3 that the dominant terms of (17) and (23) given in the ratio 27) show also a good approximation of $\mathrm{CRB}(r) / \mathrm{CRB}_{\mathrm{CG}}(r)$.

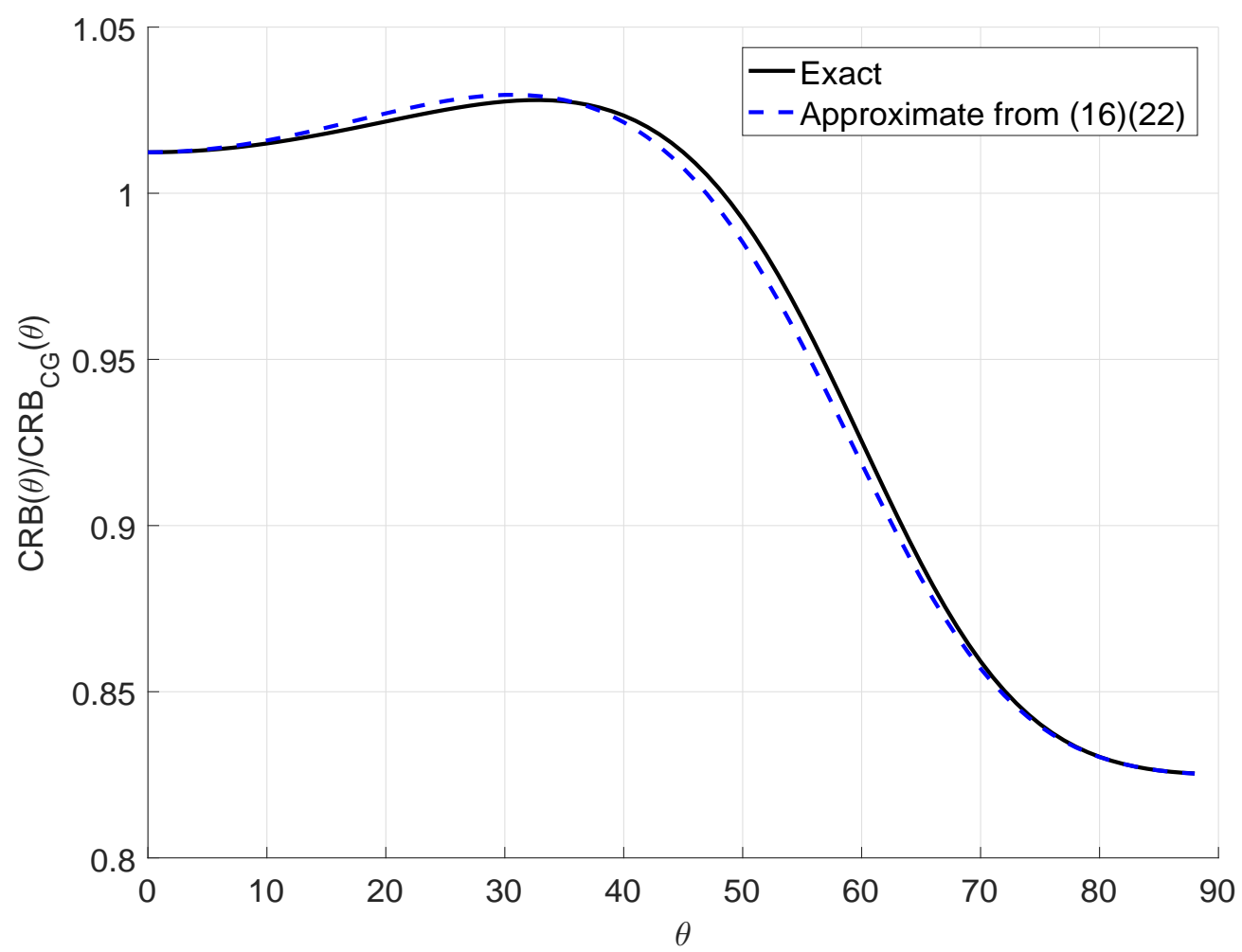

Fig.2 Exact and approximate $\operatorname{CRB}(\theta) / \operatorname{CRB}_{\mathrm{CG}}(\theta)$ as a function of $\theta$. 


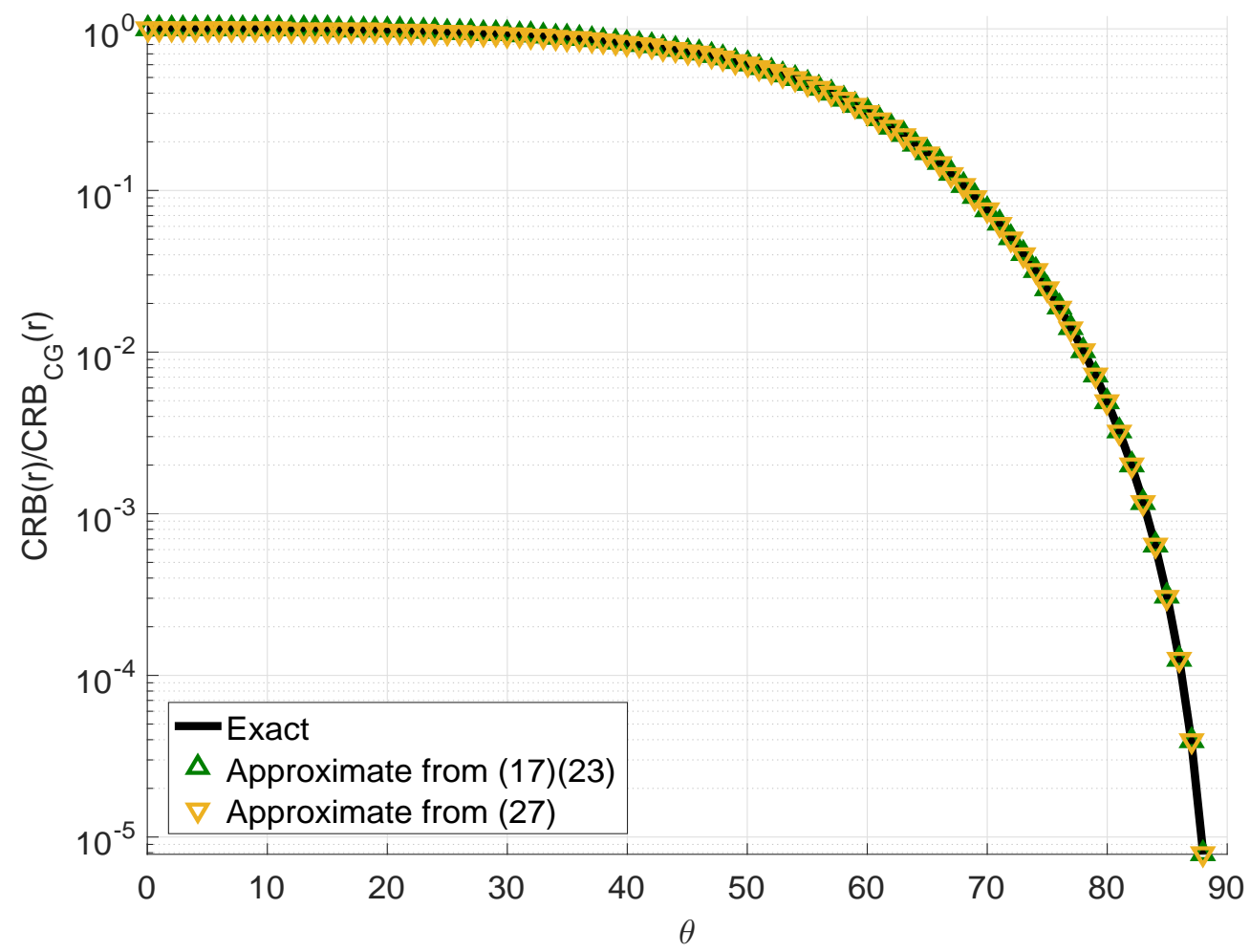

Fig.3 Exact and approximate $\mathrm{CRB}(r) / \mathrm{CRB}_{\mathrm{CG}}(r)$ as a function of $\theta$.

On the other hand, Fig.4 shows the relative CRBs on the range $\mathrm{CRB}(r) / r^{2}$ and $\mathrm{CRB}_{\mathrm{CG}}(r) / r^{2}$, assuming the same ULA used above with which we collect $N=1000$ snapshots and $\sigma_{s}^{2} / \sigma_{n}^{2}=20 \mathrm{~dB}$. We see clearly in this figure that taking into consideration the power profile allows to enlarge the domain of possible range estimation but not for broadside directions.

As a result, the localization algorithms will benefit from incorporating the power profile into the parameterization of the steering vector. This can be achieved in two ways which are outside the scope of this paper. In the first one, the localization algorithms would use the exact parameterization (2)-(4) of the steering vector. In the second one, they can use the traditional constant gain quadratic wavefront approximation model to take advantage of the low computational algorithms, but with some correction methods taking the exact parameterization model 29 . 


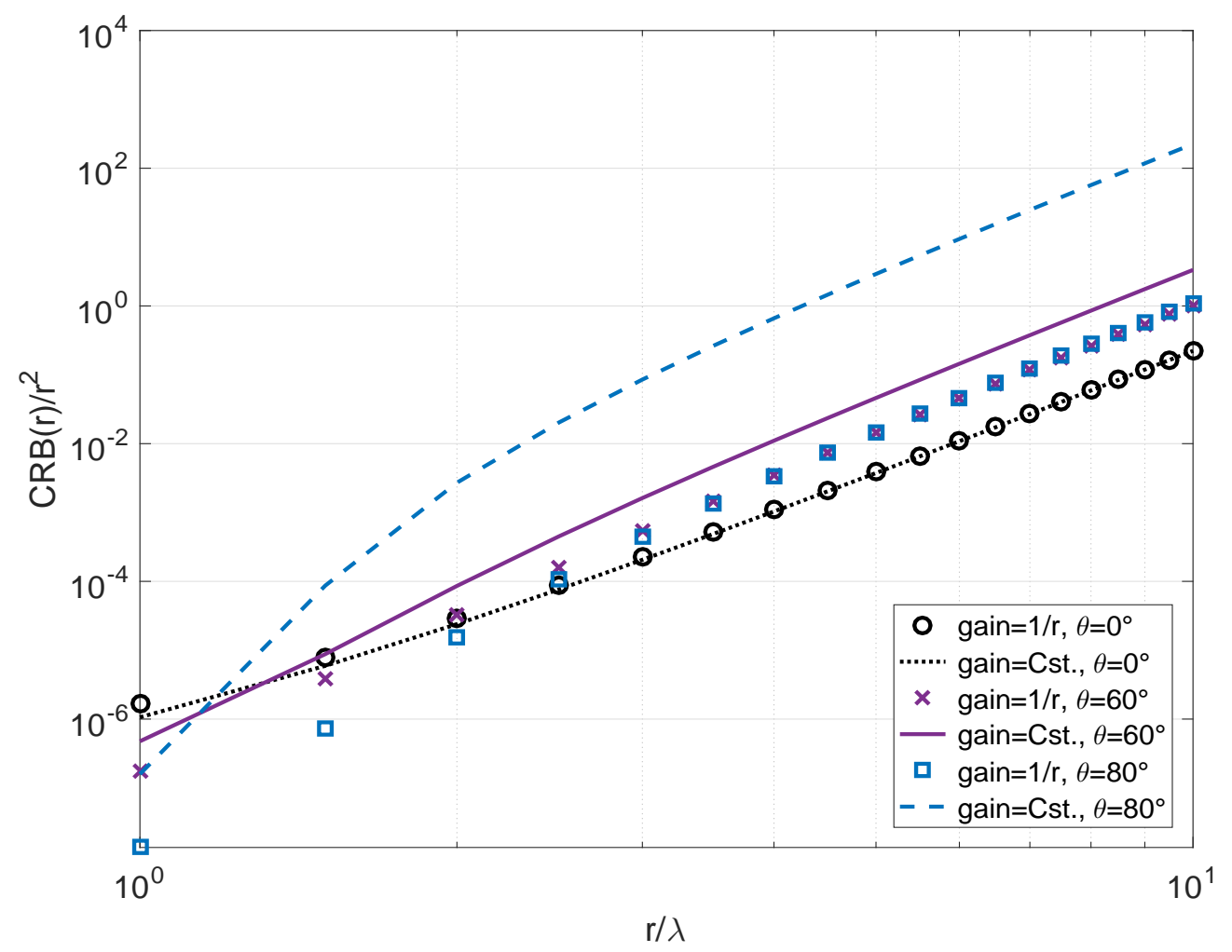

Fig.4 $\mathrm{CRB}(r) / r^{2}$ and $\mathrm{CRB}_{\mathrm{CG}}(r) / r^{2}$ with $N=1000$ and $\sigma_{s}^{2} / \sigma_{n}^{2}=20 \mathrm{~dB}$.

\section{Near-field array optimization}

When the source is in the array far-field, DOA estimation performance, as expressed in 20], depends fully on the geometric parameter $S_{2}$. But when the source is in the array near-field, additional parameters appear in (16) and (17), including the geometric parameters $\kappa$ (for DOA estimation) and both $\kappa$ and $\eta$ (for range estimation). By focusing on the dominant terms in (16) and (17), we realize that arrays with the same $S_{2}$ have the same DOA estimation performance but their range estimation performance becomes better if $\kappa$ increases. This will motivate the development of an original methodology to optimize the array geometry, one that improves its range estimation capability in the near-field and, at the same time, maintains the same DOA estimation performance, an approach followed in 30 for planar arrays with constant sensed power across its sensors.

This methodology proceeds as follows: The number $P$ of sensors is fixed. The reference antenna is the ULA with half-wavelength spacing, for which we calculate values of $S_{2}$ and $\kappa$ (renamed as $\kappa_{\text {ULA }}$ ). We are interested in identifying non-uniform centro-symmetric linear arrays associated with the same value of $S_{2}$, 
but with larger values of $\kappa$, i.e. $\kappa>\kappa_{\mathrm{ULA}}$. Such arrays satisfy:

$$
\begin{aligned}
& \operatorname{CRB}(\theta) \approx \operatorname{CRB}(\theta)_{\mid \mathrm{ULA}} \\
& \mathcal{R}_{P}(\kappa) \stackrel{\text { def }}{=} \lim _{r \rightarrow \infty} \frac{\mathrm{CRB}(r)}{\mathrm{CRB}(r)_{\mid \mathrm{ULA}}}=\frac{4 P \frac{\lambda_{\pi}^{2}}{S_{2}} \sin ^{2} \theta+\left(\kappa_{\mathrm{ULA}}-1\right) \cos ^{4} \theta}{4 P \frac{\lambda_{\pi}^{2}}{S_{2}} \sin ^{2} \theta+(\kappa-1) \cos ^{4} \theta}<1 .
\end{aligned}
$$

We note that $4 P \lambda_{\pi}^{2} / S_{2} \ll 1$ for $P>4$. Thus for values of $|\theta|$ not in the vicinity of $\pi / 2$, we have the following approximation

$$
\mathcal{R}_{P}(\kappa) \approx \frac{\kappa_{\mathrm{ULA}}-1}{\kappa-1}<1
$$

indicating better performance of arrays with $\kappa>\kappa_{\mathrm{ULA}}$.

As shown in [25], it is common that the parameter $\kappa$ lies in $[1, P / 2]$. However, it is clear that values close to $P / 2$ are to be discarded because, they correspond to a configuration where two sensors are placed at $\pm \sqrt{S_{2} / 2}$, and the remaining sensors being (almost) co-located at the centroid $O$. This is an illustration of the difficult array ambiguity problem that we tackle shortly under the following constraints: $P, S_{2}$ and $\kappa \in[1, P / 2)$ are pre-fixed based on desired near-field and far-field (DOA and range) performance. To determine positions $\left\{x_{p}\right\}_{p=1, \ldots, P}$ of the constituent sensors of the arbitrary CSLA, we are left with the following degrees of freedom: $P / 2-2$ if $P$ is even, and $(P-1) / 2-2$ if $P$ is odd.

Ambiguities occur when two steering vectors happen to be (very) close, despite referring to well separated look directions [31. One way to minimize ambiguities is to minimize the so-called relative peak sidelobe level (PSL) ratio 32] derived from the conventional array beampattern [33, which is also essentially the spatial correlation coefficient (SCC), proposed in [34. If

$$
\left[\mathbf{a}_{\mathrm{FF}}(\theta)\right]_{p} \stackrel{\text { def }}{=} \lim _{r \rightarrow \infty}[\mathbf{a}(\boldsymbol{\alpha})]_{p}=e^{\frac{i 2 \pi x_{p} \sin \theta}{\lambda}},
$$

then

$$
r_{\mathrm{PSL}} \stackrel{\text { def }}{=} \max _{\omega \text { outside the mainlobe region }}\left|\mathbf{a}_{\mathrm{FF}}^{H}(\omega) \mathbf{a}_{\mathrm{FF}}(\theta)\right|^{2} / P^{2} .
$$

The main difficulty is that $\min _{x_{1}, \ldots x_{P}} r_{\mathrm{PSL}}$, achieved under the constraints $\sum_{p=1}^{P} x_{p}^{2}=S_{2}, \sum_{p=1}^{P} x_{p}^{4}=S_{4}$ (with $S_{4}=\kappa S_{2}^{2} / P$ ) and symmetric $x_{p}$, is a non-convex minimization problem[1] In [30] we proposed an ad hoc criterion that ought to avoid concentrations of sensors in the neighborhood of the origin for large values of $\kappa$. Similarly to [30, our design technique will implement

$$
\max _{x_{1}, \ldots x_{P}}\left(\min _{1 \leq p \neq p^{\prime} \leq P}\left|x_{p}-x_{p^{\prime}}\right|\right) \text { s.t. } \sum_{p=1}^{P} x_{p}^{2}=S_{2}, \sum_{p=1}^{P} x_{p}^{4}=\kappa S_{2}^{2} / P \text { and } x_{p} \text { symmetric. }
$$

First, we note that this max-min constrained optimization (33) can be transformed into a global polynomial minimization under, both polynomial equalities and inequalities, by introducing a new decision variable $z$ as

\footnotetext{
${ }^{1}$ including for $P=6$ and 7 , for which there is a single degree of freedom, but with several local minima.
} 
follows:

$$
\begin{aligned}
& \min -z \quad \text { s.t. } z \leq 2 x_{1}, z \leq x_{p+1}-x_{p}, p=1, . ., P / 2, \sum_{p=1}^{P / 2} x_{p}^{2}=\frac{S_{2}}{2} \text { and } \sum_{p=1}^{P / 2} x_{p}^{4}=\frac{\kappa S_{2}^{2}}{2 P} \text { for Peven } \\
& \min -z \quad \text { s.t. } z \leq x_{1}, \quad z \leq x_{p+1}-x_{p}, p=1, . .,\lfloor P / 2\rfloor, \sum_{p=1}^{\lfloor P / 2\rfloor} x_{p}^{2}=\frac{S_{2}}{2} \text { and } \sum_{p=1}^{\lfloor P / 2\rfloor} x_{p}^{4}=\frac{\kappa S_{2}^{2}}{2 P} \text { for } \operatorname{Podd}(35)
\end{aligned}
$$

As such, (34) and (35) are non-convex polynomial minimizations with polynomial equalities and inequalities constraints. Following [35], these constrained minimizations can be transformed into an (often finite) sequence of convex linear matrix inequality optimization problems. These problems are solved by means of the matlab GloptiPoly utility [36] that builds and solves these convex linear matrix inequalities. By judiciously choosing the relaxation orders, we have solved our optimization problem with small relaxation order for $P=6,7,8$ and 9 sensors. As an example, Table 1 reports, for different values of $\kappa$ and the associated $\mathcal{R}_{P}(\kappa)$, the optimal sensors positions that are normalized by $S_{2}=1$, given by the criteria (33) and the relative PSL, $r_{\mathrm{PSL}}$, for $P=8$ and $\theta=0^{\circ}$. We note that our analysis is valid for a larger number $P$ of sensors, as for the polynomial approximation problem thanks to the Matlab GloptiPoly utility [36 $\left.{ }^{2}\right]$ As seen in Table 1, our objective of reducing the near-field range CRB is achieved (by up to $67 \%$ ), while ensuring arrays without ambiguity, but a tradeoff should be sought between performance improvement and the robustness to ambiguity.

\begin{tabular}{|c|c|c|c|}
\hline$\kappa$ & $\mathcal{R}_{P}(\kappa)$ & sensors positions & $r_{\mathrm{PSL}}$ \\
\hline 1.762 & 1 (ULA) & $\pm 0.0772, \pm 0.2315, \pm 0.3858, \pm 0.5401$ & 0.0525 \\
\hline 2.000 & 0.762 & $\pm 0.0699, \pm 0.2098, \pm 0.3497, \pm 0.5734$ & 0.0114 \\
\hline 2.222 & 0.624 & $\pm 0.0641, \pm 0.1922, \pm 0.3203, \pm 0.5969$ & 0.0091 \\
\hline 2.500 & 0.508 & $\pm 0.0572, \pm 0.1715, \pm 0.2858, \pm 0.6210$ & 0.0420 \\
\hline 2.857 & 0.410 & $\pm 0.0484, \pm 0.1452, \pm 0.2421, \pm 0.6465$ & 0.1398 \\
\hline 3,333 & 0.327 & $\pm 0.0358, \pm 0.1074, \pm 0.1790, \pm 0.6747$ & 0.3828 \\
\hline
\end{tabular}

Table 1 Values of $\kappa, \mathcal{R}_{P}(\kappa)$, sensors positions, $r_{\mathrm{PSL}}$.

As suggested in our analysis, for the optimized array and a source located in its near-field, reduction of the range CRB is not obtained at the expense of the DOA CRB. A confirmation can be seen in Fig.5 that exhibits the ratios $\operatorname{CRB}(\theta) /\left.\mathrm{CRB}(\theta)\right|_{\mathrm{ULA}}$ and $\mathrm{CRB}(r) /\left.\mathrm{CRB}(r)\right|_{\mathrm{ULA}}$ for a CSLA of $P=8$ sensors placed at $\pm 0.0484, \pm 0.1452, \pm 0.2421, \pm 0.6465$ with $S_{2}=1$ associated with $\kappa=2.857$ for $\theta=10^{\circ}$. We see in this figure that the CRB on the range is significantly improved without damaging the CRB on the DOA. We also observe that the values of $\operatorname{CRB}(\theta) /\left.\operatorname{CRB}(\theta)\right|_{\text {ULA }}$ and $\operatorname{CRB}(r) /\left.\operatorname{CRB}(r)\right|_{\text {ULA }}$ as functions of $r / \lambda$ are little modified for different values of $\theta \in\left[-10^{\circ},+10^{\circ}\right]$.

\footnotetext{
${ }^{2}$ The current version of Matlab GloptiPoly is able to handle our problem up to $P=39$.
} 


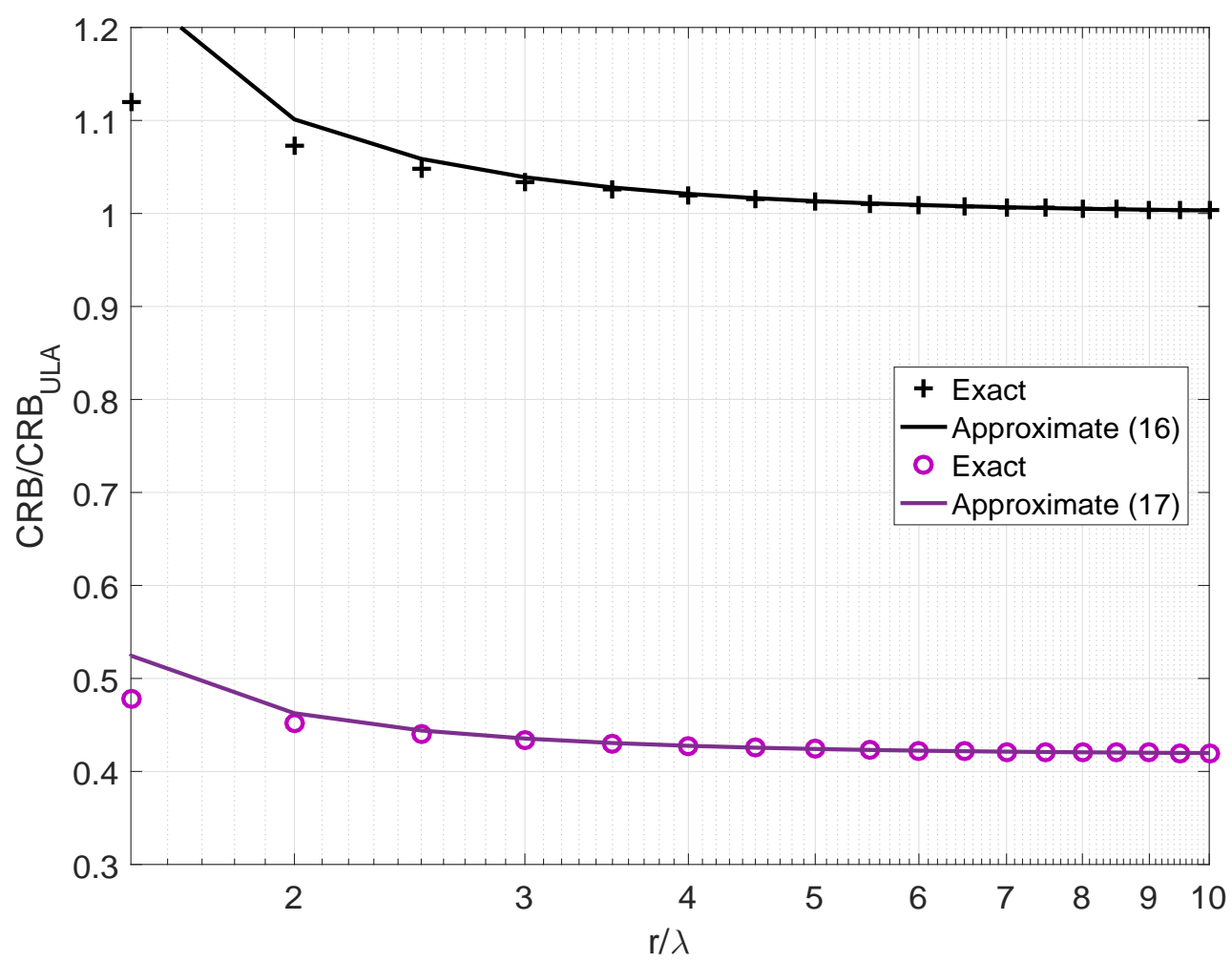

Fig.5 Exact and approximate $\operatorname{CRB}(\theta) /\left.\operatorname{CRB}(\theta)\right|_{\text {ULA }}$ and $\operatorname{CRB}(r) /\left.\operatorname{CRB}(r)\right|_{\text {ULA }}$ as a function of $r / \lambda$.

Performance in terms of errors in DOA and range have been summarized by the notion of near-field localization region (NFLR) introduced in 24. This region is based on a target estimation performance relative to the application at hand. It has been defined as the region for which the standard deviation $\sqrt{\mathrm{E}\left(\|\widehat{\mathrm{OS}}-\mathrm{OS}\|^{2}\right)}$ (where $\mathrm{OS}=r$ and $\widehat{\mathrm{OS}}$ is the estimated range) is upper-bounded by a tolerated localization error $\operatorname{Std}_{\max }$. Expressed independently of any localization algorithm, this minimum standard deviation is function of the DOA and range CRBs through the $\sqrt{r^{2} \operatorname{CRB}(r)+\operatorname{CRB}(\theta)}$ and the NFLR is defined as the region corresponding to:

$$
\sqrt{r^{2} \operatorname{CRB}(r)+\operatorname{CRB}(\theta)} \leq \operatorname{Std}_{\max }
$$

This concept of NFLR can also be used to tune the system parameters to achieve a target localization quality in the context of CSLA. In particular, this region (where the array is located in the $x$-axis) is shown for the aforementioned optimized CLSA in Fig.6 for $\sigma_{s}^{2} / \sigma_{n}^{2}=100$ and $N=100$. We see from this figure that this region is larger than its counterpart region associated with the ULA. We also see, that this region is much larger in the lateral direction than those of the ULA with constant gain. 


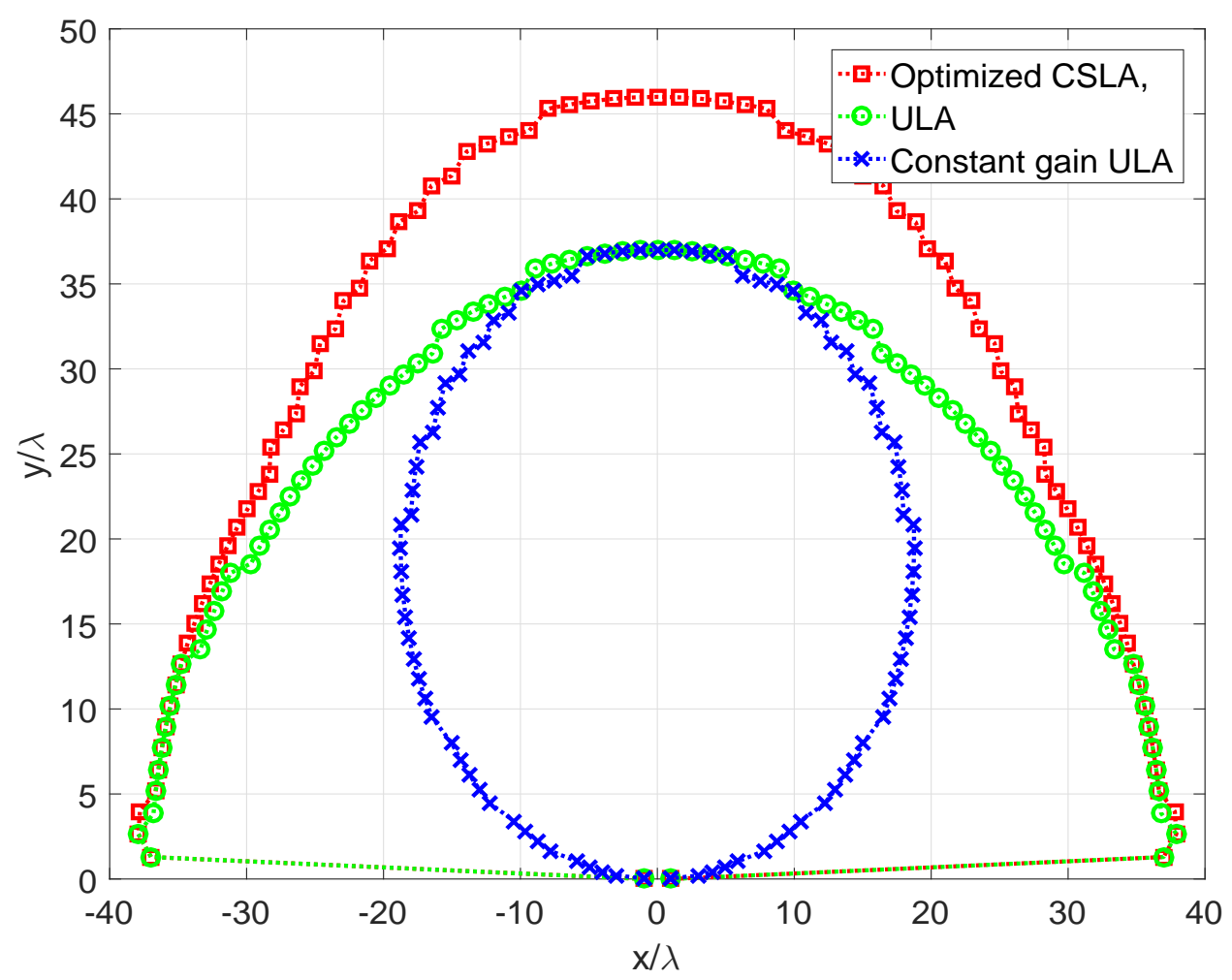

Fig.6 Comparison of NFLR of optimized CSLA with variable gain and constant gain ULA.

We note that the derivation of approximate closed-form expressions of the CRBs for multiple near-field sources under the assumptions of Section 2 seems not to be possible. However, numerical values of the deterministic $\mathrm{CRB}$ on the DOA and range can be derived from the following compact deterministic CRB expression for $Q$ parameters per source and $K$ sources straightforwardly derived from [38, th.4.1]:

$$
\mathrm{CRB}_{\mathrm{det}}(\boldsymbol{\alpha})=\frac{\sigma_{n}^{2}}{2 N}\left[\operatorname{Re}\left(\mathbf{D}^{H}(\boldsymbol{\alpha}) \boldsymbol{\Pi}^{\perp}(\boldsymbol{\alpha}) \mathbf{D}(\boldsymbol{\alpha}) \odot\left[\mathbf{1}_{Q, Q} \otimes \mathbf{R}_{s}\right]^{T}\right)\right]^{-1},
$$

where $\mathbf{D}(\boldsymbol{\alpha}) \stackrel{\text { def }}{=}\left[\mathbf{D}_{1}, \ldots, \mathbf{D}_{Q}\right]$ with $\left(\mathbf{D}_{q}\right)_{q=1, \ldots Q} \stackrel{\text { def }}{=}\left[\frac{\partial \mathbf{a}_{1}}{\partial \alpha_{q, 1}}, . ., \frac{\partial \mathbf{a}_{K}}{\partial \alpha_{q, K}}\right]$ and $\boldsymbol{\alpha} \stackrel{\text { def }}{=}\left[\alpha_{1,1}, \ldots \alpha_{1, K}, \ldots, \alpha_{Q, 1}, . ., \alpha_{Q, K}\right]^{T}$, $\boldsymbol{\Pi}^{\perp}(\boldsymbol{\alpha})$ is the so-called noise projection matrix $\mathbf{I}_{P}-\mathbf{A}(\boldsymbol{\alpha})\left[\mathbf{A}^{H}(\boldsymbol{\alpha}) \mathbf{A}(\boldsymbol{\alpha})\right]^{-1} \mathbf{A}^{H}(\boldsymbol{\alpha})$, and $\mathbf{R}_{s}$ is the covariance matrix of the sources. $\mathbf{1}_{Q, Q}$ is the $Q \times Q$ matrix of ones, $\odot$ and $\otimes$ are the Hadamard and Kronecker products, respectively. Applying (37) to the case of two equipowered uncorrelated sources of DOA $\theta_{1}$ and $\theta_{2}$ and range $r_{1}$ and $r_{2}, \boldsymbol{\alpha}=\left(\theta_{1}, \theta_{2}, r_{1}, r_{2}\right)^{T}$ and $\mathbf{R}_{s}=\sigma_{s}^{2} \mathbf{I}_{2}, \mathrm{CRB}_{\mathrm{det}}(\boldsymbol{\alpha})$ is also proportional to $c=\frac{P}{2 N} \frac{\sigma_{n}^{2}}{\sigma_{s}^{2}}$. We have noticed by many experiments, that CSLA optimized for a single source also brings some benefits for two-closely spaced sources. For example it is illustrated in Fig.7 for the optimized CLSA of 8 sensors of Fig.5 which exhibits the ratios $\operatorname{CRB}\left(\theta_{i}\right) /\left.\mathrm{CRB}\left(\theta_{i}\right)\right|_{\mathrm{ULA}}$ and $\operatorname{CRB}\left(r_{i}\right) /\left.\mathrm{CRB}\left(r_{i}\right)\right|_{\mathrm{ULA}}, i=1,2$ for $r_{1}=r_{2}$, $\theta_{1}=10^{\circ}$ and $\theta_{2}=20^{\circ}$ with respect to $r / \lambda$. We see in this figure that the optimized CLSA significantly improves both DOA and range performance for all ranges. 


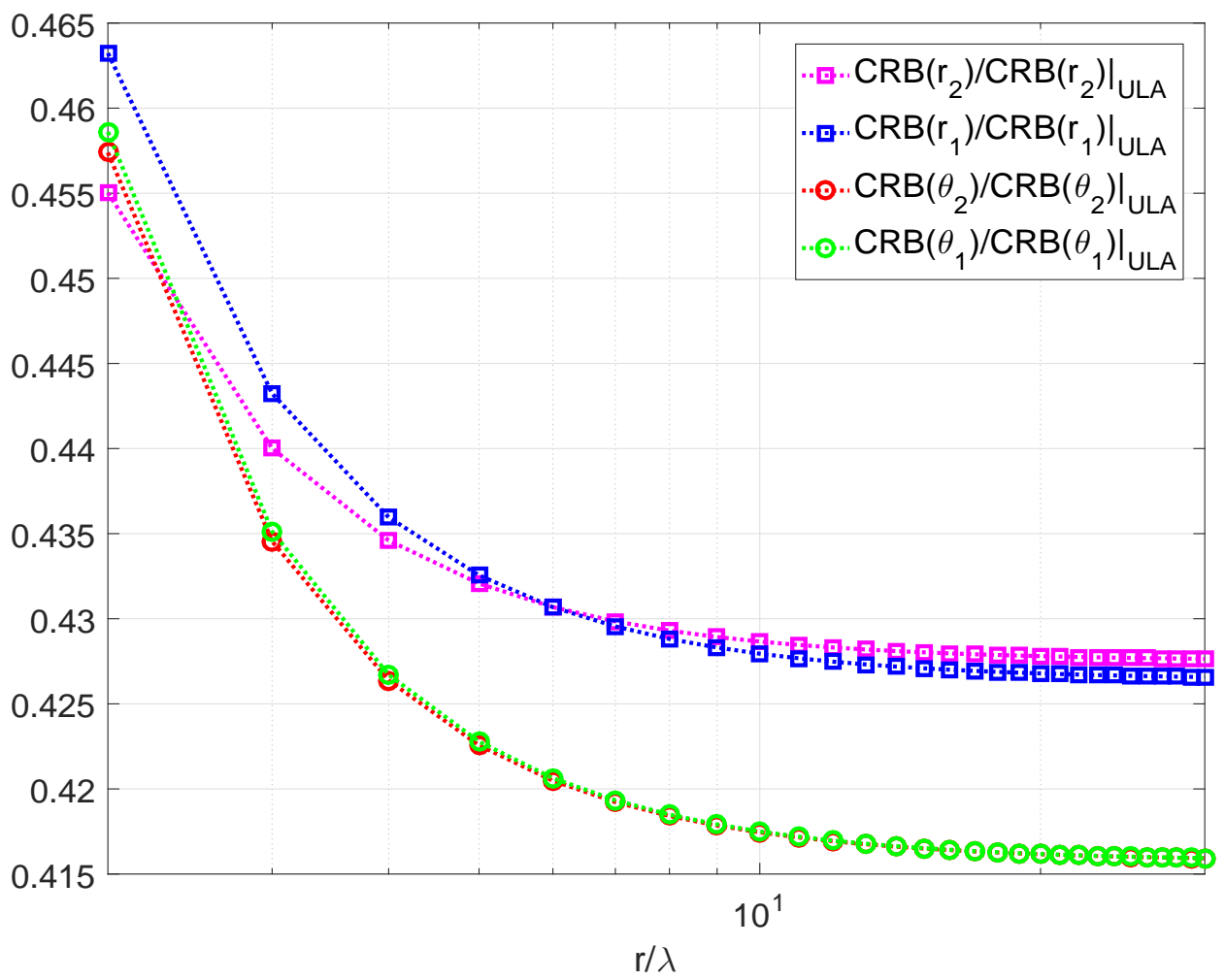

Fig.7 Exact $\operatorname{CRB}(\theta) /\left.\operatorname{CRB}(\theta)\right|_{\text {ULA }}$ and $\operatorname{CRB}(r) /\left.\operatorname{CRB}(r)\right|_{\text {ULA }}$ for two equipowered uncorrelated sources as a function of $r / \lambda$.

Otherwise we note that this improvement of performance depends on the DOA separation $\Delta \theta=\theta_{2}-$ $\theta_{1}$ as it is illustrated in Fig.8 for $r_{1}=r_{2}=9 \lambda$ and $\theta_{1}=0^{\circ}$ which shows $\operatorname{CRB}\left(\theta_{i}\right) /\left.\operatorname{CRB}\left(\theta_{i}\right)\right|_{\mathrm{ULA}}$ and $\operatorname{CRB}\left(r_{i}\right) /\left.\operatorname{CRB}\left(r_{i}\right)\right|_{\mathrm{ULA}}, i=1,2$ as a function of $\Delta \theta$. From this figure we see that the gain in performance for the estimation of the DOAs is growing monotonically when the DOA separation decreases. It practically disappears for very well separated sources $\left(\theta_{2}>80^{\circ}\right)$, similarly as in the single source case illustrated in Fig.5. In contrast, the behavior of the gain in performance for the estimation of the ranges is more involved. It keeps on being more important for very closely spaced sources. For very well separated sources $\left(\theta_{2}>80^{\circ}\right)$ the gain in performance for the estimation of $r_{1}$ is significant, similarly as in the single source case illustrated in Fig. 5 and at the opposite there is practically no gain in the estimation of $r_{2}$ because the CSLA is optimized for the estimation of the range of a single source at broadside. 


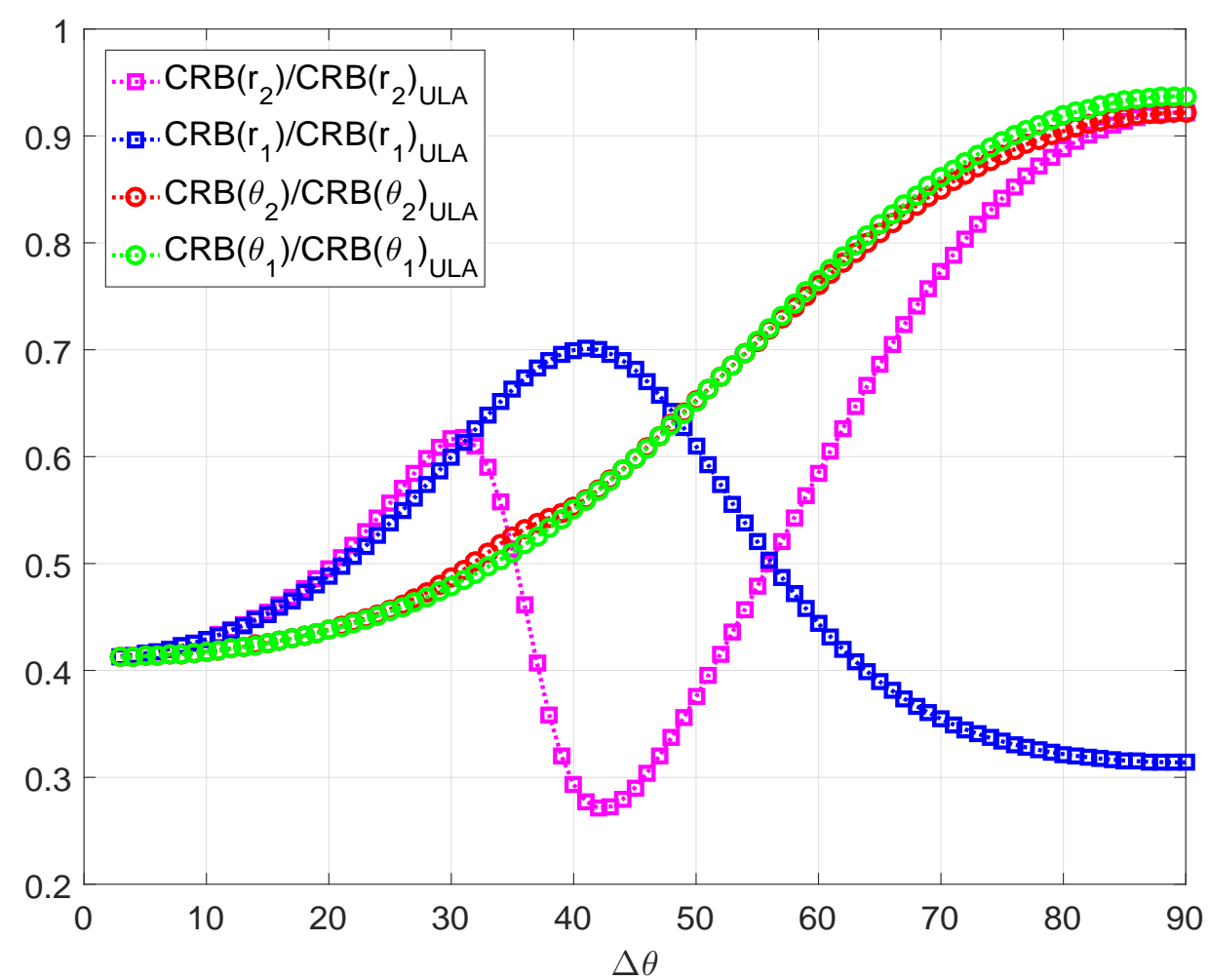

Fig.8 Exact $\operatorname{CRB}(\theta) /\left.\operatorname{CRB}(\theta)\right|_{\mathrm{ULA}}$ and $\operatorname{CRB}(r) /\left.\operatorname{CRB}(r)\right|_{\mathrm{ULA}}$ for two equipowered uncorrelated sources as a function of $\Delta \theta$ with $\theta_{1}=0$.

Finally, note that in the far-field case, the problem of optimization arises in a different way: As the CRB on the DOA only depends on the second-order moments $S_{2}$ of the positions of the sensors, the optimization consists mainly in maximizing $S_{2}$ under a constraint of non ambiguity by controlling the sidelobe level. This has been achieved by example by a selection procedure for linear and planar arrays in [37] and [32, respectively.

\section{Conclusion}

In this paper, we considered a narrow-band source located in the near-field of a linear array whose sensors are disposed symmetrically around its centroid that also serves as a reference for phase and amplitude of the received signal. For this scenario, we proposed simple and interpretable closed-form approximate expressions of the CRB on both angle and range, obtained using the exact expression of the time delay and a realistic model of power attenuation. We analyzed and compared these expressions to those not taking the power profile into account. In particular we proved that the $\mathrm{CRB}$ on the angle is little impacted by the profile of power in contrast to the CRB on the range, which is strongly reduced for not broadside directions. Consequently, the near-field localization algorithms will estimate range more accurately if the power profile 
is used in parameterization of the steering vector. Finally, these closed-form expressions are used to design nonuniform CSLAs with significantly lowered range's CRB (by as much $60 \%$ ), without deteriorating the angle's CRB, and so while taking into account ambiguity concerns of such array. Furthermore, we show by numerical experiments, that optimized CSLA for a single source also brings some benefits for two closelyspaced sources. Future research should study the improvement of performance of localization algorithms taking into account the power profile in the parameterization of the steering vectors.

\section{Appendix}

7.1. Taylor expansion of the matrix $\mathbf{F}(\boldsymbol{\alpha})$ : Proof of (11), 112) and 13)

Injecting from (4) with (3) $g_{p, 1}^{\prime} \stackrel{\text { def }}{=} \partial g_{p} / \partial \theta=\epsilon_{p} \cos \theta / \beta_{p}^{3 / 2}$ with $\epsilon_{p} \stackrel{\text { def }}{=} x_{p} / r$ into 10), we get

$$
\frac{\lambda_{\pi}^{2}}{r^{2} \cos ^{2} \theta}[\mathbf{F}(\boldsymbol{\alpha})]_{1,1}=\left(\sum_{p=1}^{P} \frac{1}{\beta_{p}}\right)\left(\lambda_{\pi}^{2} \sum_{p=1}^{P} \frac{\epsilon_{p}^{2}}{\beta_{p}^{3}}+\sum_{p=1}^{P} \frac{\epsilon_{p}^{2}}{\beta_{p}^{2}}\right)-\lambda_{\pi}^{2}\left(\sum_{p=1}^{P} \frac{\epsilon_{p}}{\beta_{p}^{2}}\right)^{2}-\left(\sum_{p=1}^{P} \frac{\epsilon_{p}}{\beta_{p}^{3 / 2}}\right)^{2} .
$$

Then using second-order expansion of $1 / \beta_{p}, 1 / \beta_{p}^{3 / 2}, 1 / \beta_{p}^{2}$ and $1 / \beta_{p}^{3}$ w.r.t. $\epsilon_{p}^{2}$, (11) is derived after cumbersome computations.

Similarly injecting from (4) and (2) with (3)

$$
\begin{aligned}
g_{p, 2}^{\prime} & \stackrel{\text { def }}{=} \partial g_{p} / \partial r=\frac{1}{r}\left(-\epsilon_{p} \sin \theta+\epsilon_{p}^{2}\right) \frac{1}{\beta_{p}^{3 / 2}}, \\
\tau_{p, 1}^{\prime} & \stackrel{\text { def }}{=} \partial \tau_{p} / \partial \theta=\left(\frac{2 \pi r}{\lambda}\right)\left(\frac{\epsilon_{p} \cos \theta}{\beta_{p}^{1 / 2}}\right) \\
\tau_{p, 2}^{\prime} & \stackrel{\text { def }}{=} \partial \tau_{p} / \partial r=\frac{2 \pi}{\lambda}\left(1+\frac{-1+\epsilon_{p} \sin \theta}{\beta_{p}^{1 / 2}}\right)
\end{aligned}
$$

into $(10)$, the expressions $(12)$ and $(13)$ are derived with the same approach.

\subsection{Taylor expansion of the CRB: Proof of (16) and (17)}

First, note that the matrix $\mathbf{F}(\boldsymbol{\alpha})$ can be written in the following form:

$$
\mathbf{F}(\boldsymbol{\alpha})=\left[\begin{array}{cc}
b_{0}^{1,1}+\frac{b_{2}^{1,1}}{r^{2}}+o\left(r^{-2}\right) & \frac{b_{3}^{1,2}}{r^{3}}+o\left(r^{-3}\right) \\
\frac{b_{3}^{1,2}}{r^{3}}+o\left(r^{-3}\right) & \frac{b_{4}^{2,2}}{r^{4}}+\frac{b_{6}^{2,2}}{r^{6}}+\frac{o\left(r^{-2}\right)}{r^{4}}
\end{array}\right],
$$

where

$$
b_{0}^{1,1}=\frac{\cos ^{2} \theta}{\lambda_{\pi}^{2}} P S_{2},
$$




$$
\begin{aligned}
\frac{b_{2}^{1,1}}{r^{2}} & =\left(\frac{\cos ^{2} \theta}{\lambda_{\pi}^{2}}\right) \frac{S_{2}\left[P \lambda_{\pi}^{2}+2 \kappa S_{2}\left(6 \sin ^{2} \theta-1\right)-S_{2}\left(1+5 \sin ^{2} \theta\right)\right]}{r^{2}}, \\
\frac{b_{3}^{1,2}}{r^{3}} & =\left(\frac{\sin \theta \cos \theta}{2 \lambda_{\pi}^{2}}\right) \frac{S_{2}\left[-2 P \lambda_{\pi}^{2}+\left(5 \kappa S_{2}-3 S_{2}\right) \cos ^{2} \theta\right]}{r^{3}}, \\
\frac{b_{4}^{2,2}}{r^{4}} & =\frac{1}{\lambda^{\prime 2}} \frac{S_{2}\left[4 P \lambda_{\pi}^{2} \sin ^{2} \theta+\left(\kappa S_{2}-S_{2}\right) \cos ^{4} \theta\right]}{4 r^{4}}, \\
\frac{b_{6}^{2,2}}{r^{6}} & =\frac{1}{\lambda_{\pi}^{2}}\left(\frac{\lambda_{\pi}^{2} S_{2}^{2}\left[\kappa\left(1-15 \sin ^{2} \theta+24 \sin ^{4} \theta\right)-\left(1-7 \sin ^{2} \theta+12 \sin ^{4} \theta\right)\right]}{r^{6}}\right. \\
& \left.+\frac{S_{2}^{3}\left[\eta\left(-5+7 \sin ^{2} \theta-99 \sin ^{4} \theta+47 \sin ^{6} \theta\right)+\kappa\left(5-49 \sin ^{2} \theta+83 \sin ^{4} \theta-39 \sin ^{6} \theta\right)\right]}{8 \operatorname{Pr}^{6}}\right) .
\end{aligned}
$$

Applying (7), where $\mathbf{F}(\boldsymbol{\alpha})$ is given by (39) allows one to obtain, after straightforward algebraic manipulations the expression of $\mathbf{F}^{-1}(\boldsymbol{\alpha})$ :

$$
\begin{aligned}
& \operatorname{CRB}(\theta)=c_{\sigma}(\boldsymbol{\alpha})\left[\mathbf{F}^{-1}(\boldsymbol{\alpha})\right]_{1,1}=\frac{c_{\sigma}(\boldsymbol{\alpha})}{b_{0}^{1,1}}\left[1-\frac{1}{r^{2}}\left(\frac{b_{2}^{1,1}}{b_{0}^{1,1}}-\frac{\left(b_{3}^{1,2}\right)^{2}}{b_{0}^{1,1} b_{4}^{2,2}}\right)+o\left(r^{-2}\right)\right] \\
& \operatorname{CRB}(r)=c_{\sigma}(\boldsymbol{\alpha})\left[\mathbf{F}^{-1}(\boldsymbol{\alpha})\right]_{2,2}=\frac{r^{4} c_{\sigma}(\boldsymbol{\alpha})}{b_{4}^{2,2}}\left[1-\frac{1}{r^{2}}\left(\frac{b_{6}^{2,2}}{b_{4}^{2,2}}-\frac{\left(b_{3}^{1,2}\right)^{2}}{b_{0}^{1,1} b_{4}^{2,2}}\right)+o\left(r^{-2}\right)\right] .
\end{aligned}
$$

Replacing the different terms $b_{k}^{i, j}$ by their values in 40 and 410 and $c_{\sigma}(\boldsymbol{\alpha})$ by $c\left(1-\frac{S_{2}}{P r^{2}}\left(1-4 \sin ^{2} \theta\right)+o\left(r^{-2}\right)\right.$ thanks to (18) and (15), the expressions of (16) and (17) are proved after tedious manipulations, where

$$
-\frac{S_{2}}{P r^{2}}\left(1-4 \sin ^{2} \theta\right)-\left(\frac{b_{2}^{1,1}}{r^{2} b_{0}^{1,1}}-\frac{\left(b_{3}^{1,2}\right)^{2}}{r^{2} b_{0}^{1,1} b_{4}^{2,2}}\right)=\left(\frac{2 \kappa S_{2}}{P}-\lambda_{\pi}^{2}\right)+g\left(\sin ^{2} \theta, S_{2}, \lambda_{\pi}^{2}, \kappa\right)
$$

with

$$
g\left(\sin ^{2} \theta, S_{2}, \lambda_{\pi}^{2}, \kappa\right)=4 P^{2} \lambda^{\prime 4}+S_{2}^{2} \kappa(13 \kappa-9) \cos ^{4} \theta+4 P S_{2} \lambda_{\pi}^{2}\left[(6-7 \kappa) \sin ^{2} \theta-(5 \kappa-3)\right]
$$

and

$$
-\frac{S_{2}}{P r^{2}}\left(1-4 \sin ^{2} \theta\right)-\left(\frac{b_{6}^{2,2}}{r^{2} b_{4}^{2,2}}-\frac{\left(b_{3}^{1,3}\right)^{2}}{r^{2} b_{0}^{1,1} b_{4}^{2,2}}\right)=h\left(\sin ^{2} \theta, S_{2}, \lambda_{\pi}^{2}, \kappa, \eta\right) .
$$

\section{References}

[1] H. Gazzah and S. Marcos, "Cramer-Rao bounds for antenna array design," IEEE Trans. Signal Process., vol. 54, no. 1, pp. 336-345, Jan. 2006.

[2] J. Li, L. Xu, P. Stoica, K. Forsythe, and D. Bliss, "Range compression and waveform optimization for MIMO Radar: a Cramer-Rao bound based study," IEEE Trans. Signal Process., vol. 56, no. 1, pp. 218-232, Jan. 2008.

[3] H. L. Van Trees, Optimum Array Processing, Part IV of Detection, Estimation, and Modulation Theory, New York, USA, Wiley, 2002.

[4] P.R.P. Hoole, Smart Antennas and Signal Processing for Communications, Biomedical and Radar Systems, Southampton, U.K.: WIT Press, 2001. 
[5] M. Haardt, M. Pesavento, F. Remer, and M. N. El Korso, "Subspace Methods and Exploitation of Special Array Structures", Electronic Reference in Signal Processing: Array and Statistical Signal Processing, vol. 3, Chapter 2.15, pp. 651-717, Academic Press Library in Signal Processing, Elsevier Ltd., 2014.

[6] W. R. Hahn, "Optimum signal processing for passive sonar range and bearing estimation," J. Acoust. Soc. Amer., vol. 58, vol. 1, pp. 201?207, 1975.

[7] G. Arslan and E.A. Sakarya, "Unified neural-network-based speaker localization technique," IEEE Trans. Neural Networks, vol. 11, pp. 997-1002, July 2000.

[8] C. M. Lee, K. S. Yoon, and K. K. Lee, "Efficient algorithm for localising 3-D narrowband multiple sources," Proc. Inst. Elect. Eng. Radar, Sonar Navig., vol. 148, pp. 23- 26, Feb. 2001.

[9] A. Sahin and E. L. Miller, "Object detection using high resolution nearfield array processing," IEEE Trans. Geosci. Remote Sens., vol. 39, no. 1, pp. 136-141, Jan. 2001.

[10] L. Giubbolini, "A multistatic microwave radar sensor for short range anticollision warning," IEEE Trans. on vehicular technology, vol. 49, no. 6, pp. 2270 - 2275, Nov 2000.

[11] C. Rascon and I. Meza, "Localization of sound sources in robotics: a review," Robot. Auton. Syst., vol. 96, pp. 184-210, 2017.

[12] A. L. Swindlelhurst and T. Kailath, "Passive direction of arrival and range estimation for near-field sources," IEEE Workshop on Spectrum Estimation and Modeling Workshop, pp. 123-128, 1988.

[13] N.L. Owsley, "Array phonocardiography," Proceedings: Adaptive Systems for Signal Processing, Communications, and Control Symposium (AS-SPCC), pp. 31-36, Oct. 2000.

[14] E. C. Fear, X. Li, S. C. Hagness, and M.A. Stuchly, "Confocal microwave imaging for breast cancer detection: Localization of tumors in three dimensions", IEEE Trans. Biomed. Eng., vol. 49, no. 8, pp. 812?822, Aug. 2002.

[15] B. Yu, "A new 2-D parameter estimation method of near-field sources based on polarization sensitive array", Computer science and network technology conference, pp. 321-324, 2012.

[16] A. J. Weiss and B. Friedlander, "Range and bearing estimation using polynomial rooting," IEEE J. Ocean. Eng., vol. 18, no. 2, pp. 130?137, Apr. 1993.

[17] N. Yuen and B. Friedlander, "Performance analysis of higher order ESPRIT for localization of near-field sources," IEEE Trans. Signal Process., vol. 46, no. 3, pp. 709-719, Mar. 1998.

[18] E. Grosicki, K. Abed-Meraim, and Y. Hua, "A weighted linear prediction method for near field source localization," IEEE Trans. Signal Process., vol. 53, no. 10, pp. 3651-3660, Oct. 2005.

[19] W. Zhi and M. Yan-Wah Chia, "Near-field source localization via symmetric subarrays," IEEE Signal Process. Letters, vol. 14, no. 6, pp. 409-412, June 2007.

[20] J. Liang and D. Liu, "Passive localization of mixed near-field and far-field sources using two-stage MUSIC algorithm," IEEE Trans. Signal Process., vol. 58, no. 1, pp. 108-120, Jan. 2010.

[21] T.J. Jung and K. Lee, "Closed-form algorithm for 3-D single source localization with uniform circular array," IEEE Trans. Antennas. Propag., vol. 13, pp. 1096-1099, June 2014.

[22] W. Zuo, J. Xin, W. Liu, N. Zheng, H. Ohmori, and A. Sano, "Localization of near-field sources based on linear prediction and oblique projection operator," IEEE Trans. Signal Process., vol. 67, no. 2, pp. 415-430, Jan. 2019.

[23] M. N. El Korso, R. Boyer, A. Renaux, and S. Marcos, "Conditional and unconditional Cramer Rao bounds for near-field source localization," IEEE Trans. Signal Process., vol. 58, no. 5, pp. 2901-2906, May 2010.

[24] Y. Begriche, M. Thameri, and K. Abed-Meraim, "Exact conditional and unconditional Cramer Rao bound for near field localization," Digital Signal Process., no. 31, pp. 45-58, August 2014.

[25] H. Gazzah and J.P. Delmas, "CRB based-design of linear antenna arrays for near field source localization," IEEE Trans. on Antenna and Propagation, vol. 62, no. 4, pp. 1965-1974, April 2014. 
[26] C. A. Balanis, Antenna Theory: Analysis and design, third edition, Wiley Interscience, 2005.

[27] P. Stoica and A. Nehorai, "Performances study of conditional and unconditional direction of arrival estimation," IEEE Trans. ASSP, vol. 38, no. 10, pp. 1783-1795, Oct. 1990.

[28] C. El Kassis, J. Picheral, and C. Mokbel, "Advantages of nonuniform arrays using root-MUSIC," Signal Processing, vol. 90, pp. 689-695, July 2009.

[29] P. Singh, Y. Wang, and P. Chargé, "A correction method for the near-field approximated model based localization techniques," Digital Signal Processing, vol. 67, pp. 76-80, 2017.

[30] J.P. Delmas, M.N. El Korso, H. Gazzah, and M. Castella, "CRB analysis of planar antenna array for optimizing near-field source localization," Signal Processing, vol. 127, pp. 117-134, March 2016.

[31] M. Gavish and A. Weiss, "Array geometry for ambiguity resolution in direction finding," IEEE Trans. Antennas Propag., vol. 44, no. 6, pp. 889-895, June 1996.

[32] X. Wang, E. Aboutanios, and M. G. Amin, "Adaptive array thinning for enhanced DOA estimation," IEEE Signal Processing letters, vol. 22, no. 7, pp. 799-803, July 2014.

[33] H. Messer, "Source localization performance and the beampattern," Signal Processing, vol. 28, no. 2, pp. 163-181, 1991.

[34] X. Wang, E. Aboutanios, M. Trinkle, and M. G. Amin, "Reconfigurable Adaptive Array Beamforming by Antenna Selection" IEEE Trans. Signal Process. vol. 62, no. 9, pp 2385-2396, May 2014.

[35] J.B. Lasserre, "Global optimization with polynomials and the problem of moments," SIAM Journal of Optimization vol. 11, no. 3, pp. 796-817, 2001.

[36] D. Henrion and J.B. Lasserre, "GloptiPoly: Global optimization over polynomials with Matlab and SeDuMi," $A C M$ Transactions on Mathematical Software vol. 29, no. 2, pp. 165-194, June 2003.

[37] V. Roy, S.P. Chepuri, and G. Leus, "Sparsity-enforcing sensor selection for DOA estimation," 5th International workshop on computational advances in multi-sensor adaptive processing (CAMSAP), Saint Martin, France, Dec. 2013.

[38] P. Stoica and A. Nehorai, "MUSIC, maximum likelihood, and Cramer-Rao bound," IEEE Trans. ASSP, vol. 37, no. 5, pp. 720-741, May 1989. 